http://dx.doi.org/10.12775/szhf.2014.005

MiroseaW Żelazny

\title{
Das Problem der Sitten im Licht der slavischen Sprachen
}

Die Anregung zu diesem Vortrag war eine Antwort, die ich vor langer Zeit auf die Frage gehört habe: „Gibt es einen Unterschied zwischen den Kantischen Begriffen „Moral“ und „Sitte“? Die Antwort lautete: „Keinen“.

Selbstverständlich ist diese Antwort schon in dem wortwörtlichen Sinn unakzeptabel. Den Unterschied zwischen den Begriffen „Moral“, „Ethik“ und "Sitte“ erklärt Kant auf der ersten Seite der Grundlegung zur Metaphysik der Sitten. Aber schenken wir unsere Aufmerksamkeit zunächst dem Titel selbst. Das Wort „Sitten“ erscheint hier im Plural, was beim Begriff „Moral“ ein sprachfremder Gebrauch wäre. „Sitten“ bedeuten eher: kurze Merksätze, die belehren, wie zu handeln ist, und nicht eine allgemeine Theorie der Moral, für Handlungen grundlegend zu sein. Die wörtliche Übersetzung des Werkes von Immanuel Kant in die polnische Sprache lautete bisher: Grundlegung zur Metaphysik der Moral, wobei man „Moral“ im Singular benutzt. Außerdem würde in der Kantischen Sprache der Satz: „....der bestirnte Himmel über mir und das Gesetz der Sitten in mir" im Grunde genommen unsinnig klingen. Das Gesetz der Sitten und das moralische Gesetz bedeuten nämlich in ethischen Schriften Kants etwas verschiedenes.

Auf den ersten Blick könnte es scheinen, es handelt sich hier um keinen wesentlichen Unterschied. Wie aber ist der Unterschied zwischen den Worten „Ethik“, „Moral“ und „Sitten“? Dieser Unterschied ist ein solcher, dass das eine aus dem Griechischen, das zweite aus dem Latein, und das dritte aus 
dem Altgermanischen stammt. In dem ursprünglichen Sinn könnten sie sich sowohl auf das Gebot beziehen "reiche nicht die Hand über die Schwelle“, das bis heute bei slavischen Völkern streng beachtet wird, als auch auf ein solches, wie: „sprich nicht schlecht über deine Mutter“, was in der ganzen Welt gilt.

In der polnischen Sprache wird die Bedeutung der beiden Sprüche abgegrenzt. Dass es in Polen zu gutem Ton gehört, bei der Begrüßung der Frau die Hand zu küssen, und dass es nichts mit Belästigung zu tun hat, bezeichnen wir als Sitte, also ein relatives kulturelles Prinzip (Volksbrauch), das gar nicht mit dem Problem in Verbindung steht, jemandem Böses anzutun. Ein ähnliches Beispiel: wenn in Indien zwei Jungen sich auf der Straße an der Hand halten, hat solch eine Geste auch nichts mit dem Geschlechtsverhalten zu tun und sie gehört zu einem sehr guten Ton. Eine andere Art dieser Bedeutung können Gebote sein, die unter slavischen Völkern beachtet werden, z.B. das erwähnte „reiche nicht die Hand über die Schwelle“, bzw. „bade nicht vor dem heiligen Johannes“, oder ,iss zu Ostern gekochte Eier“. All diese Kontexte werden in der polnischen Sprache mit dem Begriff „obyczaj“ bezeichnet, der nicht zu den Problemen strenger Moralaxiologie gehört. Wie wir am Beispiel des Volksglaubens sehen, dass die Hand über die Schwelle zu reichen, ein Unglück herbeiführen kann, bedeutet „obyczaj“ meistens keine Interpretation im Geiste der Metaphysik der Moral, sondern im Geiste der Volksfrömmigkeit, die auf eine eigene Art von dem gemeinen Verstand erfasst wird.

Ich möchte an dieser Stelle ihre Aufmerksamkeit auf eine einzigartige Perle der kantischen Weltliteratur lenken. Alle wissen wir, was die Moral Mrongovius ist - eine Reihe von Vorlesungen Kants, niedergeschrieben vom polnischen protestantischen Pastor Krzysztof Celestyn Mrongowiusz. Es wird aber oft vergessen, dass Mrongovius diese Vorlesungen über 50 Jahre vor der ersten deutschen Edition Menzers (1924) ins Polnische übersetzt und veröffentlicht hat.

Mrongovius war Kants Schüler, er besuchte seine Vorlesungen und redigierte Notizen von diesen Vorlesungen selbst, wobei er auch andere Kolleghefte in Anspruch nahm. In Königsberg studierte er Theologie und benutzte ein spezifisches Polnisch: den masurischen Dialekt. Dann kam er in Danzig mit dem kaschubischen Dialekt in Kontakt. (Überlegungen, ob Kaschuben oder Masuren Polen waren oder nicht, überlassen wir der verehrten Frau Angela Merkel. Hier könnte man sarkastisch rufen: „Es lebe der Freistaat ${ }^{1}$ Bayern!").

${ }^{1}$ Das ist die deutsche Übersetzung des Begiffs ,Republik' (im Gegensatz zum ,Königreich'). 
Für uns aber ist es von wesentlicher Bedeutung, dass sowohl die einen als auch die anderen sich eines polnischen Dialekts bedienten, der sich von der damaligen Standardsprache weit unterschied. Ähnlich war es auch mit Mrongovius, wenn er seine Predigten für Kaschuben oder Masuren hielt. Er benutzte ihre Sprache sogar dann, wenn er Kant übersetzte. Was bemerkenswert ist, er bildete dabei neue polnische philosophische Begriffe, die später wieder oft in Vergessenheit gerieten.

Dieser Aspekt seines Schaffens, d.h. die Übersetzung der Gedankens Kants in die ursprünglich slavischen Sprachen, und zwar mit Anwendung von Neologismen, hat, wie gesagt, eine sehr wesentliche Bedeutung. Seine Bedeutung betonte Kant selbst (1800) in der Nachschrift zu Mielckes littauisch-deutschem Wörterbuch: „Überhaupt, wenn auch nicht an jeder Sprache eine eben so große Ausbeute zu erwarten wäre, so ist es doch zur Bildung eines jeden Völkleins in einem Lande, z.B. im preußischen Polen, von Wichtigkeit, es im Schul- und Kanzelunterricht nach dem Muster der reinsten (polnischen) Sprache, sollte diese auch nur außerhalb Landes geredet werden, zu unterweisen und diese nach und nach gangbar zu machen: weil dadurch die Sprache der Eigenthümlichkeit des Volks angemessener und hiemit der Begriff desselben aufgeklärter wird"2.

Von welcher Wichtigkeit diese Sache ist, und welche Chancen ein solcher Vergleich eröffnet, zeigt das Schaffen von Mrongovius, dem Schüler Kants.

Im Polnischen ist heute das Wort „nie-obyczajny“ gebräuchlich, ein Wort mit einer sehr negativen Konnotation. Es betrifft unakzeptable Verhaltensweisen, meistens in Bezug auf Sex, asoziale Umgangsformen und persönliche Vorlieben. Das Wort „obyczajny“ - sein positives Antonym, ist heute nur ein sprachliches Denkmal. Es ist dagegen das Wort „obyczajowy“ in Gebrauch, das nur einen lockeren Zusammenhang mit ethischer Axiologie aufweist. Als „obyczaj“ betrachten wir es zum Beispiel, ein Brautpaar nach der Trauung mit Reis zu bestreuen. Das gilt als Glückssymbol. Dem „obyczaj" nicht nachzukommen, gilt als unhöflich, aber im Allgemeinen bildet das keine Grundlage für ein ethisches Urteil. Anders verhält es sich mit dem Wort „nie-obyczajność. Heute bedeutet es zum Beispiel eine aufdringliche Belästigung einer Frau, oder öffentliches urinieren. Früher bedeutete es ein unmoralisches Verhalten im Allgemeinen.

${ }^{2}$ Immanuel Kant, Nachschrift zu Mielckes Littauisch-deutschem Wörterbuch. Immanuel Kants Werke, hg. v. Ernst Cassirer, Bd. VIII, Berlin1922, S. 232. 
Seit dem frühen Mittelalter funktionierte in der Sprache polnischer Intellektuellen das heute geläufige Wort ,Moral'. Ins Polnische kam es durch die katholische Religion. Unter Moral verstand man eine Sammlung von Geboten, die sich auf eine spezifisch begriffene Metaphysik stützte. Es konnte z.B. die natürliche Metaphysik der Bibel gewesen sein, beispielsweise den Dekalog. Auf die polnische Kultur hat auch die Philosophie des Aristoteles' einen großen Einfluss ausgeübt, besonders in der thomistischen Interpretation. In den Universitätskreisen erfreute sich auch der Stoizismus großer Popularität.

„Obyczajność" stand im Unterschied zu ,Moral' nicht in Verbindung mit einem metaphysischen System. Sie wurde, um einen Kantischen Begriff zu gebrauchen, durch den bloßen Verstand vollzogen, den man für den gesunden Menschenverstand (common sense) hielt. Sie betraf also, wenn wir uns auf die Einteilung von den Anfangsseiten der Grundlegung zur Metaphysik der Sitten berufen, den empirischen Teil der Metaphysik der Moral (Ethik), der an der gleichen Stelle sogar als anthropologisch bestimmt wurde.

Mrongovius übersetzt die Kantischen „Sitten“ mit „obyczajnośćc, was heißt: allgemein übliche Prinzipien, die vorschreiben, wie man sich zu verhalten habe. Auf welche Art und Weise hat dieser hervorragende Kenner mit der noch im 19. Jhd. archaischen slavischen Sprache diesen Begriff verstanden? Und wie haben ihn seine Gemeindemitglieder, Masuren und Kaschuben, vestanden? Versuchen wir uns auf die Etymologie zu stützen.

Der polnische „obyczaj“ besteht aus zwei Teilen: Das Wort „oby“, das sich nur sehr schwer ins Deutsche übertragen lässt, bedeutet so viel wie: es möge geschehen. Das altslavische „czaj“ bedeutet dagegen: lauern. Wenn ein Fuchs zum Beispiel sich nicht verraten will, weil er im Versteck auf ein Opfer wartet, sagen wir: er lauert. Auch ein Trupp Soldaten im Hinterhalt lauert. Und ein Politiker lauert, wenn er bei der Debatte seine Meinung verbirgt, um im günstigen Augenblick seinen Gegner zu schlagen.

Aber das ist nur die erste Bedeutungsschicht. Es ist allgemein bekannt, dass im Russischen „czaj“ Tee bedeutet, also ein heißes Getränk. Dies ist aber nicht die ursprüngliche Bedeutung, welche auf die Zeremonie des Teetrinkens zurückgeht, die nach bestimmten Gesetzen verlief. In Russland, genauso wie in England, trank man Tee zu bestimmter Uhrzeit oder in einer bestimmten Situation. Man brauchte für diese Zeremonie einen Samowar mit Kohle aus geeigneter Holzart. Es ging um das Aroma des Getränks. Der Tee befand sich in Gläsern auf schweren Metalluntersätzen. Der Sinn davon war, dass der Trinkende sich allein auf die Tätigkeit des Trinkens konzentriert. Zucker hat man in der Form eines - wie man sagt - Zuckerhuts serviert. Für einen 
kultivierten Russen wäre es ein Akt der Grobheit, Zucker aus der Zuckerdose, oder in Würfeln zu benutzen. War jemand zur „czaj“-Zeremonie zugelassen, musste er ein Zuckerstück mit einem speziellen Messer bearbeiten können. Das war eine viel kompliziertere Fertigkeit, als z.B. mit chinesischen Stäbchen zu essen. Wer dessen nicht mächtig war, sollte sich sozusagen nicht in die Gesellschaft drängen. Das losgelöste Zuckerstück warf man nicht ins Glas, sondern man legte es unter die Zunge. Zum Tee servierte man eine Spezialität: geröstete Walderdbeeren.

Das Ziel der Sitte des „czaj“-Trinkens lässt sich sehr leicht bemerken. Es ist einer uralten Tradition entsprungen. In Tibet kennt man Gebetswerkzeuge, die sogenannte Gebetmühle, oder ein anderes, ein Zylinder mit Gebeten, den man zum Drehen bringt. Es muss gedreht werden, ohne dass man den Zylinder berührt, und das gelingt nur, wenn man sich ganz auf die Bewegung konzentriert. Wenn unsere Gedanken woanders hin schweifen, mahnt uns ein Mechanismus, der die Bewegung des Zylinder beeinträchtigt.

Die Bezeichnung für die „czaj“ - Zeremonie stammt aus dem altslavischen „czaić się“. Es bedeutet: auf alle unnötigen körperlichen Bewegungen verzichten, nichts Unnötiges erzählen, sich von keiner überflüssigen Erregung hinreißen lassen. Und als Belohnung ein leibliches Vergnügen genießen können. Im allgemeinsten jedoch: innerhalb einer Gemeinschaft zwischenmenschliche Kommunikation aufbauen.

Wir möchten es betonen: in diesem Sinn, d.h. als zeremonielles „Sichselbst-zurücknehmen", die Kommunikation und Verabredung zwischen Personen herzustellen, ist in vielen verschiedenen, voneinander unabhängigen Kulturen bekannt. Und es ist auch in den gegenwärtigen Kulturen üblich. Zum Beispiel vor Gericht müssen die jeweiligen Anwälte in spezifischer ritueller Bekleidung antreten. Die Gerichtsverhandlung muß nach bestimmten Verfahrensregeln durchgeführt werden. Unabhängig von den Umständen sollte sie in Würde, mit Objektivität und mit gegenseitigem Respekt aller Teilnehmer stattfinden. Der Pole würde sagen: „obyczaj“, der Deutsche: „Sitte“, aber für einen Polen heißt der (linguistische!) Grundzug jener Sitte: lauern ${ }^{3}$. Die Gerichtsverhandlung nennt man auf polnisch auch: „posiedzenie“, was man ins Deutsche als Sitzung übersetzt. Das polnische „po-siedzenie“ und die deutsche Sitzung, stammen von dem gleichen uralten Wort: sitzen. Da-

\footnotetext{
${ }^{3}$ Wäre es auch möglich, lieber Miroslaw, dieses „lauern“ mit „"auf-der-Hut-sein“, „Obachtgeben“ oder „aufmerksam-sein“ zu übersetzen?
} 
mit kann man die Genealogie von deutschen „Sitten“, russischem „czaj“ und polnischem „oby-czaj“ verknüpfen.

Diese Betrachtungen zeigen deutlich, was für Grundlagen maßgebend waren, wenn man ein Werturteil nach dieser Instanz fällte, das man gesunden Menschenverstand nennt. Was verbindet z.B. das Gebot: lass die Frau zuerst einsteigen, wenn du mit einer Straßenbahn fährst, mit dem Gebot: du sollst die körperliche Unantastbarkeit der Person beachten. Der Pole würde sagen, dass die erste Situation sich auf „obyczaj“ bezieht, also das Gebot gilt durch die allgemeine, konventionelle Sitte. Aber es muss nicht in allen möglichen Situationen gelten, weil sich zum Beispiel erklärte Feministinnen nicht gefallen lassen können, nur deshalb bevorzugt zu werden, weil sie Frauen sind. Ähnlich könnte es zum Beispiel bei einer deutschen Studentin Anstoß erregen, ja, sie könnte sich belästigt fühlen, wenn ein älterer polnischer Professor ihre Hand küssen würde. Dagegen wäre das für die Mehrzahl polnischer Studentinnen ein nettes Ereignis. Dieser Typ der Gebote, von „obyczaj“ abgeleitet, ist ein Effekt von „przyczajenie“, also ein Effekt der Gewohnheit. Nach diesem Prinzip sollte niemandem Vorwürfe machen werden, dass er nicht auf russische Art Tee trinken gelernt hat, aber er sollte sich auch nicht der Gesellschaft aufdrängen, in der man es pflegt.

Dagegen ist, wie im Fall der deutschen Studentin mit dem polnischen Handkuss, die Grenze der herkömmlichen Konvention überschritten und das kann sogar als sehr unzumutbar empfunden werden. Das wäre der klassische Fall eines kulturellen Missverständnisses. Wir haben hier mit dem Fall zu tun, den der Pole als moralisches Übel bezeichnen würde.

Den Kantischen Begriff "Sitten“ verstehen also die Polen mit zwei Worten. Das erste ist „obyczaj“, also ,Gewohnheit” oder ,Konvention'. Hier wird betont, dass eine Tat, die für unkultiviert oder auch nur unhöflich gehalten wird, nicht zur eigenen Kultur, oder Sitte passt. Das zweite ist „Moral“, und es bezieht sich auf etwas, was eine metaphysische Erklärung beansprucht. Ein Merkmal polnischer Kultur ist es, eine Situation nicht zuzulassen, in der die koventionelle, herkömmliche Sitte es beansprucht, ein metaphysisches Postulat zu sein. Diese Tendenz lässt sich übrigens in vielen Kulturen beobachten, bloß in den slavischen Sprachen wurde sie durch eine sehr originelle Terminologie hervorgehoben, die auf etymologische Bedeutungsvarianten zurückgeht.

Der bisherige Teil meines Vortrags stellt den Versuch dar, die Moral Mrongovius im Geiste der polnischen Textfassung zu interpretieren, ein Ergebnis der Arbeit von Mrongovius. Ich hatte nur den empirischen Teil der „Meta- 
physik der Sitten“ im Blick. In der polnischen Interpretationstradition würde das Gebot: küsse nicht die Hand einer Frau, weil es sie erschrecken könnte, nicht zu dem gehören, was Kant als „Metaphysik der Sitten“ bezeichnet hat. Und zwar aus einem sehr einfachen Grund: um festzustellen, ob in einem konkreten Fall ein Handkuss ein Akt der Höflichkeit oder Unhöflichkeit ist, ist keine Metaphysik notwendig. Es genügt das Wissen über die Konvention und das Bewusstsein, dass die Person, die sich so verhalten hat, es mit gutem Willen gemacht hat. Anders ist es mit der Situation bewandt, wenn im Verhalten einer Person böser Willen erkennbar ist, z.B. sie provoziert absichtlich unangenehme Situationen, um sich daran zu vergnügen. Solch ein Verhalten nennen wir unmoralisch.

Die von Mrongovius definierten Auslegungen der Kantischen Probleme, sowohl in Deutsch, als auch in Polnisch, sind beide auf ihre eigene Art mit einigen Mängeln behaftet. Der polnische Begriff „obyczajność” würde ein Verhalten umfassen, die Kraft der Konventionen als übel gelten. Nur einige unter ihnen kann man in dem Sinn für übel halten, den die Kantischen Sitten festlegen. Es wären solche, deren bösen Charakter von der Metaphysik bestimmt wird. Die Schuld dafür, solche Taten begangen zu haben, trägt der Mensch aber auch dann, obwohl sein Wille meistens stark durch „obyczaj“ determiniert wird. Dazu gehören solche üblen Taten, wie z.B. unverheiratete schwangere Frauen zu verspotten. Moral ist für einen Polen das zweite Glied der Kantischen „Sitten“ und bezieht sich auf Taten, die deutlich aus bösem Willen verübt wurden. Polnische Begriffe sind in diesem Fall detaillierter, es ist aber unmöglich mit ihnen solch einen Termin wie ,Sittengesetz' zu formulieren, der den materialen Teil der Metaphysik der Moral anbetrifft. Sprechen wir aber über ihren formalen (apriorischen) Teil, der sich auf Moralgesetz bezieht, dann gibt es natürlich keinen Zusammenhang zwischen polnischer Konvention und dem System Kants. Es sei denn, wir stellen die Suche nach Analogie zu den Systemen von Aristoteles' oder Thomas von Aquin zur Diskussion, was an polnischen Universitäten die Oberhand erhält. Aber das wäre eine sehr ferne Analogie.

Der Terminus „Ethik“ dagegen, von Kant als Wissenschaft begriffen, die den formalen und den materialen Teil der Lehre über axiologische Probleme vereinigt, hat in der polnischen philosophischen Sprache eine ähnliche Bedeutung wie bei Kant und wie auch im deutschen Denken im allgemeinen. Diese Interpretation gilt nicht z.B. für polnische Übersetzungen der Schriften Hegels, in denen die Gegenüberstellung der Begriffe ,Moral' - ,Sitten' noch einen anderen Sinn hat. 
Dieser kurze Vortrag war nur ein Versuch, die Aufmerksamkeit auf zwei wesentliche Probleme zu lenken. Das erste betrifft den Kern des Unterschiedes zwischen Begriffen „Moral“ und „Sitten“ bei Kant. Das zweite betrifft eine sehr wichtige Frage, die in der erwähnter Nachschrift zum Litauisch-Deutschem und Deutsch-Litauischem Wörterbuch in der Redaktion Miehlers angeschnitten wird. Kant unterstreicht hier eine wichtige Tatsache: auf die Sprachen zurückzugreifen, die in der ursprünglicheren Form erhalten geblieben sind, kann eine sehr wichtige Erfahrung für den Umgang mit gegenwärtigen europäischen Sprachen mit sich bringen.

Kant selbst, der sicherlich in Königsberg mit der polnischen Sprache in Berührung kam, hat mindestens einmal solch eine Erfahrung gemacht. In seinen Vorlesungen zur Anthropologie führt er nämlich die Etymologie des Wortes „Willkür“ auf das polnische „kur“ zurück, was „Hahn“ bedeutet. Wenn wir wissen, dass dieses Haustier auch in Deutschland ein Symbol des Eigensinns ist, verstehen wir diese Analogie gut, obwohl es fragwürdig bleibt, ob sie sprachlich-wissenschaftlich begründet ist.

In dieser Hinsicht ist die Moral Mrongovius, die die Vorlesungen Kants in die ursprüngliche und stellenweise regionale polnische Sprache übersetzt, ein echter Schlussstein in der Kantischen Architektonik. Es geht nicht einfach um die Übersetzung, sondern um eine andere Fassung von Kants Vorlesungen, die von Mrongovius - dem Autor der Notizen - verfasst wurde. Die beiden Fassungen also, die polnische und die deutsche, sind auf ihre eigene Art authentisch. Aber die Tatsache, dass das Polnische hier eine Basisform besitzt, die von der philosophischen Hauptsprache des 19. Jhds. weit entfernt lag, bringt es mit sich, dass es hier - indem wir von der deutschen Literatursprache zum ursprünglichen Polnisch übergehen, welches zu noch mit dem masurischen und dem kaschubischen Dialekt vermengt wurde - zu einer natürlichen phänomenologischen Reduktion kommt. Das Polnische stellt hier dem Deutschen Fragen, die vielleicht in anderen Umständen nicht zum Vorschein kämen.

\section{Abstract \\ The Problem of Morality (Sitten) in the Light of Slavonic Languages}

The article is an attempt to answer the frequently formulated question: Is there a difference between the notions of Moral and Sitte? The most common answer is: these notions were used synonymously by Kant, so expressing the difference between 
them - if it were to be emhasized - is difficult for translators of Kant's works. Some important intuitions about how to express the nuances of Kantian terminology in Polish can be found in the letters of Christopher Celestine Mrongowius, a Polish student of Kant, translator, writer and the author of famous collections of notes on morality made during Kant's lectures.

Key words: Immanuel Kant, morality, ethics, history of philosophy, the problems of translation of philosophical texts 